\title{
Transit timing, depth, and duration variation in exoplanet TrES-2?
}

St. Raetz ${ }^{1}$, G. Maciejewski ${ }^{1}$, M. Mugrauer ${ }^{1}$, T.O.B. Schmidt ${ }^{1}$, T. Roell ${ }^{1}$, T. Eisenbeiss ${ }^{1}$, A. Berndt ${ }^{1}$, M.M. Hohle ${ }^{1,2}$, Ch. Ginski ${ }^{1}$, R. Errmann ${ }^{1}$, M. Seeliger ${ }^{1}$, Ch. Adam ${ }^{1}$, T. Pribulla ${ }^{1,3}$, N. Tetzlaff ${ }^{1}$, M. Vaňko ${ }^{3}$, J. Koppenhoefer ${ }^{4}$, M. Raetz ${ }^{5}$ and R. Neuhäuser ${ }^{1}$

${ }^{1}$ AIU Jena, Schillergässchen 2-3, 07745 Jena, Germany

${ }^{2}$ MPE, Giessenbachstrasse, 85748 Garching, Germany

${ }^{3}$ Astronomical Institute, Slovak Academy of Sciences, Slovakia

${ }^{4}$ University Observatory Munich, München, Germany

${ }^{5}$ Private observatory Raetz, Herges-Hallenberg, Germany

\begin{abstract}
We report on our ongoing search for timing, duration, and depth variations in the exoplanet TrES-2. In Raetz et al. (2009) we already presented ten different transits obtained at the University Observatory Jena. Between November 2008 and August 2010 twelve additional transits could be observed. The timing, depth and duration of each individual event was analyzed and is presented here.
\end{abstract}

\section{Introduction}

The transit event is - in a first approximation - a periodic phenomenon. In a system where a known planet transits its host star, a second planet in that system can cause the time between transits to vary. The presence of additional bodies in transiting systems cannot only lead to variations in the transit times, but also to variations of depth, durations, and light curve shapes. Transit Timing, Transit Depth, and Transit Duration Variation permits to identify additional bodies and also to characterize exoplanetary systems.

\section{Instruments and Observations}

Most observations were carried out at the University Observatory Jena. For our transit observations we use the $25 \mathrm{~cm}$ Cassegrain auxiliary telescope equipped with the

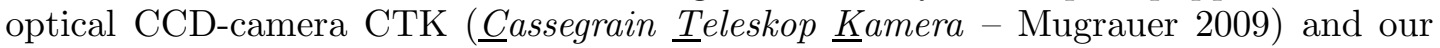

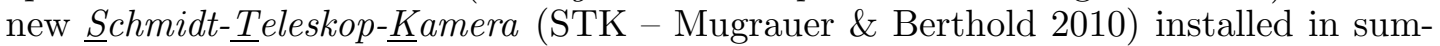
mer 2009 in the Schmidt focus of the $90 \mathrm{~cm}$ telescope. For our TrES-2 observations, started in March 2007, we used 54 nights since March 2007 to August 2010. All TrES-2 observations were taken in $V$-, $R$-, or $I$-band with exposure times between 20 and $60 \mathrm{~s}$.

\section{Transit Timing Variations}

Altogether we could obtain 22 different transits of TrES-2 between March 2007 and August 2010. In Raetz et al. (2009) we already presented ten transit observations 
which resulted in redetermining the ephemeris. We do not see any evidence for transit timing variation. Our twelve additional transits are entirely consistent with the updated ephemeris (see Fig. 1a), as well as with the most recent analysis of Kepler's shortcadence photometry by Kipping \& Bakos (2010).

a.)

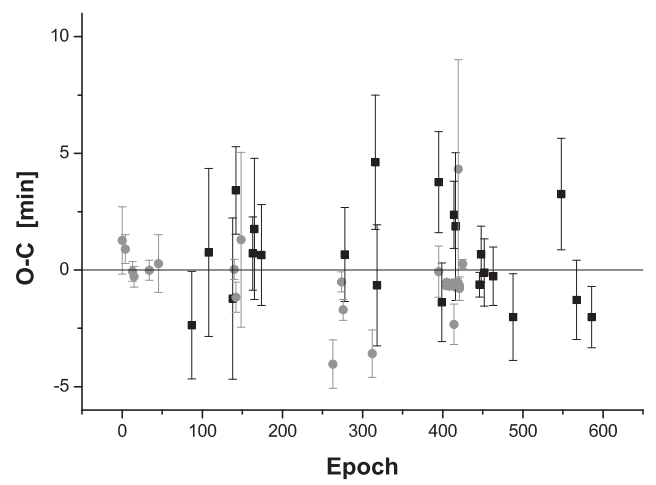

b.)

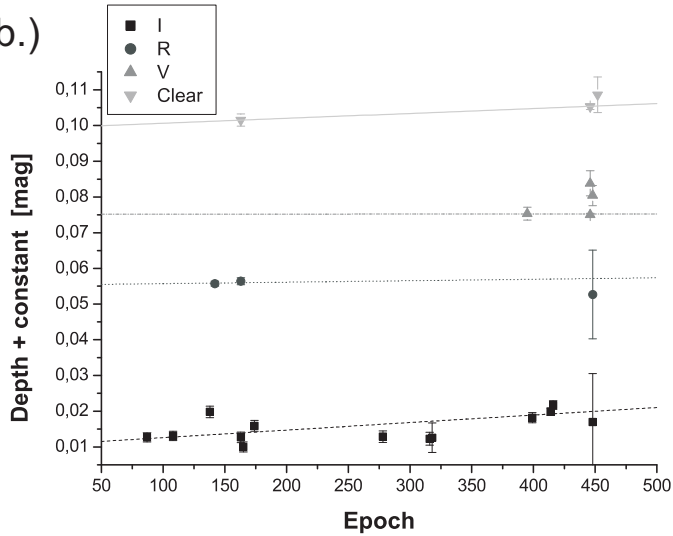

Figure 1: a.) O-C for TrES-2. Grey circles - literature data. Black squares - this work. The black line give our updated ephemeris b.) Depth over Epoch split in the different filters.

\section{Transit Depth Variations}

We analyzed the transit depth of TrES-2 for all events observed at the University Observatory Jena. We found that there is a slight positive trend which is visible in all 4 bandpasses used by us (see Fig. 1b). One finds the biggest slope in $I$-band while the $V$-band exhibits the smallest slope. It seems that the transit of TrES-2 is is increasing over time.

\section{Transit Duration Variations}

While analyzing the duration of all our transit observations of TrES-2 we also could not find any evidence for transit duration variation. Again this result is consistent with the findings of Scuderi et al. (2010) and of Kepler (Kipping \& Bakos 2010).

\section{Discussion and Outlook}

We observed several transits of the known exoplanet TrES-2 at the University Observatory Jena. We analyzed these altogether 22 transits individually and determined the transit time, depth, and duration. We do not find any evidence for transit timing and duration variations. These results are consistent with the findings of Scuderi et al. (2010) and of Kepler (Kipping \& Bakos 2010). For the transit depth we found that there is a slight positive trend. It seems that the transit depth increases over time. We will continue observing TrES-2 to check for timing, depth, and duration variation for the next few years to decades. 
Acknowledgements. SR, TR thank DFG in program NE 515/32-1. SR, GM, TP acknowledge support from the EU. GM, RN thank DAAD PPP-MNiSW project 50724260-2010/20011. CG, RN, AB thank the DFG for support in programs NE 515/30-1, 23-1 and KR 2164/8-1, respectively. MMH thank DFG in SFB-TR 7. NT thanks Carl-Zeiss-Stiftung.

\section{References}

Kipping, D. M., \& Bakos, G. Á. 2010, arXiv:1006.5680

Mugrauer, M. 2009, AN, 330, 419

Mugrauer, M., \& Berthold, T. 2010, AN, 331, 449

Raetz, S., et al. 2009, AN, 330, 459

Scuderi, L. J., Dittmann, J. A., Males, J. R., Green, E. M., \& Close, L. M. 2010, ApJ, 714, 462 